\title{
Glucose-6-phosphate- dehydrogenase is also increased in erythrocytes from adolescents with Down syndrome
}

\author{
Francisco J. Ordonez', Manuel Rosety-Plaza' and Manuel Rosety- \\ Rodriguez ${ }^{2}$
}

\author{
${ }^{1}$ Human Anatomy and Embryology Department, School of Medicine, University of Cadiz, Spain \\ ${ }^{2}$ Medicine Department, School of Medicine, University of Cadiz, Spain
}

\begin{abstract}
For some time it has been claimed that trisomic cells are more sensitive to oxidative stress since there is an imbalance in hydrogen peroxide metabolism due to an increase in superoxide dismutase (SOD) catalytic activity. We designed the present study to assess activity levels of antioxidant enzymes [superoxide dismutase (SOD), glutathione peroxidase (GPX), catalase (CAT) and glucose-6-phosphate-dehydrogenase (G6PDH)] in erythrocytes in 31 male adolescents with Down syndrome (mean age $16.3 \pm 1.1$ ). An increase of $35.2 \%, 15.3 \%$ and $14.9 \%$ in the catalytic activity of SOD, GPx and G6PDH respectively was observed in male adolescents with Down syndrome compared to age-matched controls. For CAT, a slight increase of $6.0 \%$ was also found. It is concluded that our data are consistent with previous evidence of the existence of oxidative stress in individuals with Down syndrome as revealed by significantly enhanced activities of SOD and GPx. The most striking feature was that G6PDH, in contrast to CAT, presented a similar behaviour. Further studies are required to identify other antioxidant enzymes in red blood cells as well as in white blood cells in order to increase the range of potential bioindicators of oxidative stress.
\end{abstract}

Keywords: Down syndrome, erythrocyte, antioxidant enzymes, glucose-6-phosphate-dehydrogenase

\section{Introduction}

Since it has been widely accepted that reactive oxygen species (ROS) can activate signalling processes and induce cytotoxicity in many disorders, there has been intense ongoing research on this topic, mainly regarding the role of the antioxidant system.
In recent years it has been claimed that trisomic cells are more sensitive to oxidative stress since there is an imbalance in hydrogen peroxide metabolism (Carratelli et al., 2001). Thus, individuals with Down syndrome present an increase in SOD1 catalytic activity that produces an excess of $\mathrm{H}_{2} \mathrm{O}_{2}$ that reacts with superoxide anion $\left(\mathrm{O}_{2}^{-}\right)$, producing hydroxyl radical $\left(\mathrm{OH}^{-}\right)$, which is one of the most active radical oxygen species (Chance, 1979). This 
F.J. Ordonez, M. Rosety-Plaza and M. Rosety-Rodriguez - Glucose-6-phosphate-dehydrogenase increase in erythrocytes

fact is of particular interest since oxidative stress has been proposed as a pathogenic mechanism of atherosclerosis, cell aging, carcinogenic events, immunological default, cataract formation and neurologic disorders in individuals with Down syndrome (Cengiz et al., 2002; Ianello et al., 1999; Pastore et al., 2003).

Erythrocytes are susceptible to oxidative damage as a result of the high polyunsaturated free fatty acid content of their membrane and the high cellular concentrations of oxygen and haemoglobin, a potentially powerful promoter of oxidative processes (Tan \& Yeung, 1995). To date, major antioxidant enzymes such as superoxide dismutase (SOD), glutathione peroxidase (GPx) and catalase (CAT) have been found increased in erythrocytes of individuals with Down syndrome (Groner et al., 1994; Muchova et al., 2001).

Glucose-6-phosphate dehydrogenase (G6PDH), an enzyme of the pentose phosphate pathway, is essential to control the intracellular reductive potential by increasing the intracellular glutathione level, which in turn decreases the amount of ROS (Lukaszewicz-Hussain, 2003). Consequently, it is well known that in some tissues (e.g., liver, adipose, lung and proliferating cells), G6PDH activity is increased by oxidative stress (Kletzien et al., 1994).

For the reasons already mentioned the present study was designed to assess erythrocyte glucose-6-phosphatedehydrogenase activity in male adolescents with Down syndrome in order to analyse its role as a minimally invasive bioindicator of oxidative damage.

\section{Material and method}

In the present study, 31 male adolescents with Down syndrome (mean age $16.3 \pm 1.1$ ) were enrolled. None of them suffered acute medical problems at the time and they had not taken part in any physical activity program in the last six months. The control group was comprised of 17 healthy siblings of monitored persons with Down syndrome (mean age $16.6 \pm 1.3$ ). Written informed consent was obtained from all their parents.

Venous blood samples were taken from the antecubital vein with suitable vacutainers with EDTA as anticoagulant. The basal venous blood was obtained from all the participants in this study on the morning after 12 hours of overnight fasting. In all cases blood samples were taken according to the principles of the Helsinki declaration.

Erythrocyte pellets were obtained from 1 $\mathrm{mL}$ of fasting venous blood by centrifuga- tion at $500 \mathrm{~g}$ for $10 \mathrm{~min}$ at room temperature immediately after the blood was drawn. The plasma and buffy layer were then removed, and the erythrocytes were washed three times in a $9 \mathrm{~g} / \mathrm{L} \mathrm{NaCl}$ solution. Lysed erythrocytes were prepared by putting cells through three freeze-thaw cycles in dry ice and by the addition of five volumes of ice-cold distilled water. Cell membranes were removed by centrifugation, and the supernatant was frozen at -20 ${ }^{\circ} \mathrm{C}$ until the determination antioxidant enzyme activities (Pastor et al., 1998).

The activities of superoxidase dismutase (SOD, E.C. 1.15.1.1 McCord \& Fridovich, 1969), glutathione peroxidase (GPX, E.C. 1.11.1.9 Sies, 1986), catalase (CAT, E.C. 1.11.1.6 Beutler, 1975a) and glucose-6-phosphate dehydrogenase (G6PDH, E.C. 1.1.1.49 Glock \& McLean, 1953) were determinated in the supernatant of erythrocyte haemolysates. In this respect it should be mentioned that enzyme activities were related to cell haemoglobin content and expressed as $\mathrm{U}$ or $\mathrm{mU} / \mathrm{g}$ haemoglobin.

Results were expressed as mean \pm SD. The statistical analysis of data was performed using Student's $t$-test for unpaired data. The significance of the changes observed was ascertained at $p<0.05$.

\section{Results}

Antioxidant catalytic activity results are listed in Table 1. An increase of $35.2 \%, 15.3 \%$ and $14.9 \%$ in the catalytic activity of SOD, GPx and G6PDH respectively were observed in male adolescents with Down syndrome compared with control subjects. For CAT, an increase of $6.0 \%$ was also found.

The activities of superoxide dismutase (SOD), glutathione peroxidase (GPX) and glucose-6-phosphate dehydrogenase $(\mathrm{G} 6 \mathrm{PDH})$, were increased significantly in adolescents with Down syndrome when compared with controls $(p<0.05)$. Catalase activity was also increased in

\section{Table 1. Catalytic activity of the superoxide dismutase (SOD), glutathione peroxidase (GPx), catalase (CAT) and glucose-6- phosphate-dehydrogenase (G6PDH) antioxidant enzymes in erythrocytes of adolescents with Down syndrome and in age- matched controls}

\begin{tabular}{|l|c|c|c|c|}
\hline & D.S. $(\mathrm{n}=31)$ & Ctrls. $(\mathrm{n}=17)$ & Dif. $(\%)$ & $p$ \\
\hline SOD (U/g Hb) & $679.0 \pm 82$ & $502.0 \pm 70$ & 35.2 & $0.019^{*}$ \\
\hline GPx (U/g Hb) & $24.8 \pm 3.1$ & $21.5 \pm 2.9$ & 15.3 & $0.030^{*}$ \\
\hline CAT (U/g Hb) & $1607.0 \pm 284$ & $1516.0 \pm 262$ & 6.0 & 0.151 \\
\hline G6PDH (mU/g Hb) & $12.3 \pm 1.2$ & $10.7 \pm 1.0$ & 14.9 & $0.038^{*}$ \\
\hline
\end{tabular}

Note: D.S.: Down syndrome individuals; Ctrls: Controls;

Dif.: Difference; $p$ : statistical $p$ value. Results are expressed as mean \pm SD.

* The significance of the changes observed was ascertained at $p<0.05$. 
the Down syndrome group but the differences were not statistically significant $(p>0.05)$.

\section{Discussion}

Several disorders have been related to oxidative stress in people with Down syndrome and consequently it is of interest to examine the role of antioxidant defences in general and enzymatic systems in particular. In this respect, the importance of blood cells is increasing since they are cells rich in antioxidant enzymes and they may reflect changes in the catalytic activity of these enzymes in other less accessible tissues (Richards et al., 1998).

As was hypothesised we found significantly higher catalytic activity levels for SOD in erythrocytes from adolescents with Down syndrome compared with controls (Groner et al., 1994; Muchova et al., 2001). The increase in SOD enzyme activity is due to an excess of genetic information given that superoxide dismutase (SOD) gene is localised to chromosome 21q22.1 (Gulesserian et al., 2004).

SOD catalyses the dismutation of the superoxide anion $\left(\mathrm{O}_{2}^{-}\right)$to $\mathrm{H}_{2} \mathrm{O}_{2}$ and then, in a second step, glutathione peroxidase (GPx) and catalase (CAT) convert hydrogen peroxide $\left(\mathrm{H}_{2} \mathrm{O}_{2}\right)$ to water. Accordingly, it is widely accepted that the activity of the first-step (SOD) and second-step (GPx, CAT) antioxidant enzymes must be balanced to prevent cell damage by oxidative stress (Crosti et al., 1989). Consequently and in agreement with Pastor et al., (1998) it is conceivable that any excess $\mathrm{H}_{2} \mathrm{O}_{2}$ produced by the action of SOD would induce a significant increase in the catalytic activity of GPx. On the other hand we have not observed significant changes in the activity of CAT as was reported previously (Brugge et al., 1999; Muchova et al., 2001).

In animal cells, and especially in human erythrocytes, the principal antioxidant enzyme for the detoxification of $\mathrm{H}_{2} \mathrm{O}_{2}$ has long been considered to be GPx, as catalase has much lower affinity for $\mathrm{H}_{2} \mathrm{O}_{2}$ than does GPx. Glutathione peroxidases (GPx) are selenium-dependent enzymes that can decompose $\mathrm{H}_{2} \mathrm{O}_{2}$ and various hydroand lipid peroxides by the glutathione redox cycle. In this reaction reduced glutathione $(\mathrm{GSH})$ is used as a cosubstrate to metabolise $\mathrm{H}_{2} \mathrm{O}_{2}$ to $\mathrm{H}_{2} \mathrm{O}$ and oxidised glutathione (GSSG). Oxidised glutathione (GSSG) can be reduced back to GSH by the enzyme GSH reductase (GR), a reaction requiring NADPH regenerated by glucose 6-phosphate dehydrogenase (G6PDH) (Jollow \& Mcmillan 2001; Lukaszewicz-Hussain, 2003).

We found that the catalytic activity of G6PDH was also significantly increased when compared with controls. This fact may be of interest for researchers since it is accepted that G6PDH fuels the first reaction of the pentose phosphate pathway whose biological functions are to synthesise ribose as the sugar components of nucleotides and to maintain a continuous supply of NADPH as the obligatory substrate for the glutathione system protecting cells against oxidative stress (Lukaszewicz-Hussain, 2003; Sailaja et al., 2003).

An observation one can make when reviewing the literature is that the present series $(n=31)$ was clearly larger than those reported in previous studies in individuals with Down syndrome (Pastor et al., 1998; Groner et al., 1994). Taking into account our results as well as those reported previously in the literature we may conclude that erythrocyte antioxidant enzymes (SOD, GPx and (AT) in adolescents with Down syndrome are significantly higher than controls. In addition, these adolescents presented a significant increase in glucose6-phosphate-dehydrogenase catalytic activity. It should also be emphasised that these antioxidant alterations may be determined by means of a minimally invasive technique.

Further studies are required to identify other antioxidant enzymes in red blood cells as well as in white blood cells in order to increase the range of potential bioindicators of oxidative stress.

\section{Correspondence}

M. Rosety-Rodriguez • Medicine Department, School of Medicine, University of Cadiz, Pza. Fragela s/n 11003 Cadiz, Spain • Tel. +34 956015201 • Fax. +34 8561720 $05 \bullet$ e-mail: manuel.rosetyrodriguez@uca.es

\section{Acknowledgment}

This study was supported by a grant from Centro Andaluz de Medicina del Deporte (No Ref. CTD 2003-062/01). Resolución de 11 de diciembre de 2003 de la Consejería de Turismo y Deporte. Junta de Andalucía.

\section{References}

Beutler, E., (1975). Catalase. In E. Beutler (Ed.), Red Cell Metabolism. A manual of biochemical methods. New York: Grune and Straton. pp. 89-90.

Brugge, K., Nichols, S., Sayito, T. \& Trauner, D. (1999). Correlations of glutathione peroxidase activity with memory impairment in adults with Down syndrome. Biological Psychiatry, 46, 1682-1689.

Carratelli, M., Porcaro, L., Ruscica, M., De Simone, E., Bertelli, A.A. \& Corsi, M.M. (2001). Reactive oxygen metabolites and prooxidant status in children with Down's syndrome. International Journal of Clinical Pharmacology Research, 21, 79-84. 
F.J. Ordonez, M. Rosety-Plaza and M. Rosety-Rodriguez - Glucose-6-phosphate-dehydrogenase increase in erythrocytes

Cengiz, M., Seven, M. \& Suyugul, N. (2002). Antioxidant system in Down syndrome: a possible role in cataractogenesis. Genetic Counseling, 13, 339-342.

Crosti, N., Bajer, J., Gentile, M., Resta, G. \& Serra, A. (1989). Catalase and glutathione peroxidase activity in cells with trisomy 21. Clinical Genetics, 36, 107-116.

Chance, B., Sies, H. \& Boveris, A. (1979). Hydroperoxide metabolism in mammalian organs. Physiological Review, 59, 527-605.

Glock, G.E. \& McLean, P. (1953). Further studies on the properties and assay of glucose-6-phosphate dehydrogenase and 6-phosphogluconate dehydrogenase of rat liver. The Biochemical Journal, 55, 400-408.

Groner, Y., Elroy-Stein, O., Abraham, K.B., Schickler, M., Knobler, H., Minc-Golomb, D., Bar-Peled, O., Yarom, R. \& Rotshenker, S. (1994). Cell damage by excess CuZnSOD and Down's syndrome. Biomedicine and Pharmacotherapy, 48, 231-240.

Gulesserian, T., Seidl, R., Hardmeier, R., Cairns, N. \& Lubec, G. (2001). Superoxide dismutase SOD1, encoded on chromosome 21, but not SOD2 is overexpressed in brains of patients with Down syndrome. Journal of Investigative Medicine, 49, 41-46.

Iannello, R.C., Crack, P.J., de Haan, J.B. \& Kola, I. (1999). Oxidative stress and neural dysfunction in Down syndrome. Journal of Neural Transmission Supplementum, 57, 257-267.

Jollow, D.J. \& McMillan, D.C. (2001). Oxidative stress, glucose-6-phosphate dehydrogenase and the red cell. Advances in Experimental Medicine and Biology, 50, 595-605.

Kletzien, R.F., Harris, P.K. \& Foellmi, L.A. (1994). Glucose-6-phosphate dehydrogenase: a "housekeeping" enzyme subject to tissue-specific regulation by hormones, nutrients, and oxidant stress. The FASEB Journal, 8, 174-181.

Lukaszewicz-Hussain, A. (2003). The role of glutathione and glutathione-related enzymes in antioxidative process. Medycyna Pracy, 54, 473-479.

McCord, J.M. \& Fridovich, I. (1969). Superoxide dismutase. An enzymic function for erythrocuprein (hemocuprein). The Journal of Biological Chemistry, 244, 6049-6055.

Muchova, J., Sustrova, M., Garaiova, I., Liptakova, A., Blazicek, P., Kvasnicka, P., Pueschel, S. \& Durackova, $Z$. (2001). Influence of age on activities of antioxidant enzymes and lipid peroxidation products in erythrocytes and neutrophils of Down syndrome patients. Free Radical Biology and Medicine, 31, 499-508.

Pastor, M.C., Sierra, C., Dolade, M., Navarro, E., Brandi, N., Cabre, E., Mira, A. \& Seres, A. (1998). Antioxidant enzymes and fatty acid status in erythrocytes of Down's syndrome patients. Clinical Chemistry, 44, 924-929.

Pastore, A., Tosí, G., Gaeta, L.M., Giannotti, A., Bertini, E., Federici, G., Digilio, M.C. \& Piemonte, F. (2003). Glutathione metabolism and antioxidant enzymes in children with Down syndrome. The Journal of Pediatrics, 142, 583-535.

Richards, R.S., Roberts, T.K., Dunstan, R.H., McGregor, N.R. \& Butt, H.L. (1998). Erythrocyte antioxidant systems protect cultured endothelial cells against oxidant damage, Biochemistry and Molecular Biology International, 46, 857-865.

Sailaja, Y.R., Baskar, R. \& Saralakumari, D. (2003). The antioxidant status during maturation of reticulocytes to erythrocytes in type 2 diabetics, Free Radical Biology and Medicine, 35, 133-139.

Sies, H. (1986). Biochemistry of oxidative stress. Angewandte Chemie International Edition in English, 25, 1058-1071.

Tan, W. \& Yeung, E.S. (1995). Simultaneous determination of enzyme activity and enzyme quantity in single human erythrocytes, Analytical Biochemistry, 226, 74-79. 